\title{
A Non-invasive Real-Time Methodology for the Quantification of Antioxidant Properties in Coffee During the Roasting Process Based on Near-Infrared Spectroscopy
}

\author{
Tiago A. Catelani ${ }^{1} \cdot$ Ricardo N. M. J. Páscoa ${ }^{2} \cdot$ João Rodrigo Santos ${ }^{3}$. \\ Leonardo Pezza $^{1}$ • Helena R. Pezza ${ }^{1}$ José L. F. C. Lima ${ }^{2}$ João A. Lopes ${ }^{4}$
}

Received: 14 June 2016 / Accepted: 30 November 2016 / Published online: 13 December 2016

(C) Springer Science+Business Media New York 2016

\begin{abstract}
This paper describes the development of a real-time method based on near-infrared spectroscopy (NIRS) for determination of total antioxidant capacity (TAC) and total phenolic content (TPC) in coffee during the roasting process. The real-time non-invasive monitoring procedure involved pointing a diffuse reflectance probe directly at the roasting chamber through a glass window in order to monitor the roasting process. The figures of merit of the chemometric models to estimate TAC and TPC showed selectivity values higher than $12 \%$ and determination coefficients $\left(R_{\mathrm{P}}^{2}\right)$ above 0.90 . The TAC and TPC profiles during the roasting procedure are discussed in terms of the antioxidant compounds likely to be present in green and roasted coffee. NIRS was found to be a satisfactory real-time tool for monitoring the content of antioxidant compounds in coffee during the
\end{abstract}

Electronic supplementary material The online version of this article (doi:10.1007/s11947-016-1843-6) contains supplementary material, which is available to authorized users.

João Rodrigo Santos

jrodrigosantos@fc.up.pt

1 Institute of Chemistry, State University of São Paulo - UNESP, R. Prof. Francisco Degni 55, P.O. Box 355, Araraquara, SP, Brazil

2 REQUIMTE/LAQV - Departamento de Ciências Químicas, Laboratório de Química Aplicada, Faculdade de Farmácia, Universidade do Porto, Porto, Portugal

3 REQUIMTE/LAQV - Departamento de Química e Bioquímica, Faculdade de Ciências, Universidade do Porto, Porto, Portugal

4 Research Institute for Medicines (iMed.ULisboa), Faculdade de Farmácia, Universidade de Lisboa, Lisbon, Portugal roasting process, complementing other established procedures.

Keywords Near-infrared spectroscopy $\cdot$ Coffee roasting · Real-time monitoring · Total antioxidant capacity · Total phenolic content

\section{Introduction}

Interest in the development of new and higher quality coffee beverages has led to the marketing of single origin and/or blended coffees that possess distinct organoleptic characteristics. An additional focus concerns coffees rich in bioactive compounds, especially in relation to the high intrinsic antioxidants content of this beverage (Carlsen et al. 2010). Antioxidant compounds have been extensively studied due to their potential beneficial health effects, since they help to protect against harmful reactive species (Lee et al. 2004). The most important coffee compounds, in terms of antioxidant properties, are phenolics, melanoidins, and caffeine. The first group includes hydroxycinnamic acids such as caffeic and ferulic acids (Gallardo et al. 2006), their esters, and quinic and chlorogenic acids (Moreira et al. 2005). As a result of their similar bioactivity and chemical properties, chlorogenic acids and hydroxycinnamic acids have been grouped together as CHAs (Budryn et al. 2015). Isoflavones are also included within the phenolics group but occur at lower concentrations in coffee beans, contributing less than $0.1 \%$ on a dry weight basis (dwb) (Alves et al. 2010). Overall, phenolic compounds comprise ca. $10 \% \mathrm{dwb}$ of green coffee and decrease during the roasting process. Caffeine (Devasagayam et al. 1996), which shows no significant concentration change during roasting, accounts for ca. 1-2\% dwb. Lastly, an additional antioxidant source has been attributed to melanoidins (Borrelli et al. 2002; 
Delgado-Andrade et al. 2005), which are formed in Maillard reactions during the roasting process and may be present at up to $25 \% \mathrm{dwb}$ in roasted coffee.

Quantification of the total antioxidant capacity (TAC) in coffee has been performed using assays with $2,2^{\prime}$-azinobis (3-ethylbenzothiazoline-6-sulfonic acid) (ABTS) (PérezMartínez et al. 2010; Sánchez-González et al. 2005) and 2, 2-diphenyl-1-picrylhydrazyl (DPPH) (Delgado-Andrade et al. 2005). The determination of total phenolic compounds (TPCs) is typically performed by the Folin-Ciocalteu procedure (Pérez-Martínez et al. 2010). These methodologies require an extraction step prior to the analysis. Therefore, determination of the antioxidant profile (TAC and TPC) during the roasting process can only be performed off-line, based on discrete sampling. Antioxidants have been analyzed in studies of coffee brewing procedures (Pérez-Martínez et al. 2010; Sánchez-González et al. 2005; Vignoli et al. 2011), coffee varieties (Vignoli et al. 2011), degree of roast (DelgadoAndrade et al. 2005; Morais et al. 2009; Vignoli et al. 2011), and bioavailability of the compounds (Budryn et al. 2015).

The main characteristics of near-infrared spectroscopy (NIRS), namely fast response, no sample pre-treatment, and ease of handling, make this technique especially appealing for use in the coffee industry. NIRS has been used successfully for quantification of chemical compounds in coffee samples (Pizarro et al. 2004, 2007), discrimination between Arabica and Robusta species (Esteban-Díez et al. 2007), and prediction of sensory properties and degree of roast (Esteban-Díez et al. 2004; Ribeiro et al. 2011). Recently, in a pioneering approach, NIRS was employed for in situ prediction of titratable acidity (Santos et al. 2016a) and sucrose concentration (Santos et al. 2016b) during coffee roasting processes. The potential to accompany chemical reactions during coffee roasting enables more efficient implementation of corrective procedures, better understanding of the roasting process, and, most importantly, improvement of the general quality of the coffee product.

This work describes the use of NIRS with a diffuse reflectance probe for the real-time in situ monitoring of TAC and TPC during the roasting process. Prediction models for realtime quantification of antioxidant compounds are presented, and the influence of different roasting procedures and coffee varieties is discussed.

\section{Experimental}

\section{Coffee Samples and Roasting Procedure}

Four coffees from different origins were purchased locally for use in the experimental assays: two Arabica coffees originating from Brazil and Timor and two Robusta coffees originating from India and Uganda.
The coffee roasting was performed with a bench coffee roaster (Model KN-8828B-2K, Hottop USA) that featured a frontal glass observation window (through which the in-line NIR spectra were collected), a rotatory roasting drum for homogeneous roasting of the coffee batch, and a sampling door through which coffee sampling could be performed during the roasting process. The bench coffee roaster performs automatically the roasting process after previous setting of the heating power (that defines the temperature-increase/time ratio) and fan speed (which aids normalizing the roasting process). Two different roasting procedures were employed, designated as "fast roast" (FR) and "slow roast" (SR), based on a previous work (Santos et al. 2016a). In FR, coffee samples were heated continuously up to $225^{\circ} \mathrm{C}$ during approximately $18 \mathrm{~min}$; in SR, coffee samples were heated continuously up to $195^{\circ} \mathrm{C}$ for approximately $25 \mathrm{~min}$. For FR and SR modes, the heating power was set to 7 and 5, respectively, and the fan's speed was set to $25 \%$ according to the roaster's parameter scales. Batches of approximately $300 \mathrm{~g}$ of green coffee from the different origins were weighed out and roasted following the FR and SR procedures. Samples weighing approximately $5 \mathrm{~g}$ were collected every $3 \mathrm{~min}$ in order to comprise different roasting intensities during each roasting process. Sampling was performed by manually pulling the knob that opened the sampling door. The door was kept open for ca. $1 \mathrm{~s}$ during each sampling, avoiding significant perturbation of the roasting process. The collected samples were placed in borosilicate flasks. A total of 56 samples were collected (4 origins $\times(6$ FR samples +8 SR samples $))$. The experimental conditions selected focus on different raw materials and roasting policies, aiming at generating a fair number of distinct coffee samples for the predictive model construction.

\section{Determination of Total Antioxidant Capacity and Total Phenolic Content}

\section{Reagents and Solutions}

All reagents were analytical grade and were used without further purification. Ethanol (Panreac, 121086.1212, $\geq 99.8 \%$ ) and water with resistivity $>18 \mathrm{M} \Omega \mathrm{cm}$, obtained from a Milli-Q deionizer, were used to prepare the solutions.

For the TAC assays, ABTS (Fluka, 11557, $\geq 99 \%$ ) and potassium persulfate $\left(\mathrm{K}_{2} \mathrm{~S}_{2} \mathrm{O}_{8}\right.$, Sigma-Aldrich, 21622-4, $\left.\geq 99 \%\right)$ were prepared at concentrations of 14 and $4.9 \mathrm{mmol} \mathrm{L}^{-1}$, respectively. Equal volumes of these reagents were mixed for formation of the ABTS ${ }^{+}$radical. This solution was left over$\operatorname{night}(>12 \mathrm{~h})$, followed by preparation of standard solutions in ethanol/water medium $(1: 1, v / v)$ in order to achieve an absorbance value of $0.900 \pm 0.020$ (at $734 \mathrm{~nm}$ ). Trolox (SigmaAldrich, 238813, $\geq 97 \%$ ) was used as the standard in the determination of total antioxidant capacity. A stock solution was prepared daily at a concentration of $780 \mu \mathrm{mol} \mathrm{L}^{-1}$ in ethanol/ 
water solution $(1: 1, v / v)$. Working standard solutions of Trolox were prepared by appropriate dilutions of the stock solution.

For the determination of TPC, Folin-Ciocalteu reagent (Sigma-Aldrich, F9252) and sodium carbonate (Fluka, $713510, \geq 99 \%$ ) were prepared in aqueous medium at concentrations of 3:10 $(v / v)$ and $9 \%(w / v)$, respectively. Gallic acid (Fluka, 48630, $\geq 98 \%$ ) was used as standard. An aqueous stock solution was prepared daily at a concentration of ca. $200 \mathrm{mg} \mathrm{L}^{-1}$. Working standard solutions of gallic acid were prepared by appropriate dilutions of the stock solution.

\section{Antioxidant Compounds Extraction Procedure}

Prior to extraction, the moisture contents of all the collected samples were determined according to the standard methodology (ISO 11294:1994). The extraction method used was as described previously (Páscoa et al. 2013): an accurately weighed out portion (ca. $50 \mathrm{mg}$ ) of each previously milled sample was extracted with $20.0 \mathrm{~mL}$ of ethanol/water solution $(1: 1, v / v)$ for $5 \mathrm{~h}$, using an orbital shaker (Model 3005, GFL) at $300 \mathrm{rpm}$. The selected extraction solution composition ensured the solubilization of both lipophilic and hydrophilic compounds, as well as the interaction of antioxidants with radical species (Gökmen et al. 2009; Mussatto et al. 2011; Pérez-Jiménez and Torres 2011). This procedure was performed in duplicate for all the collected samples. The solutions were left to stand until a clear supernatant was obtained and were then decanted. Finally, appropriate dilutions of the clear ethanolic solutions were made prior to the determination of TAC and TPC.

\section{Total Antioxidant Capacity of Roasted Coffee}

The total antioxidant capacity of the collected coffee samples was determined according to the procedure described by Páscoa et al. (2013). Aliquots $(150 \mu \mathrm{L})$ of coffee extract (previously diluted 40 -fold) were mixed with $150 \mu \mathrm{L}$ of ABTS $^{+}$solution in 96-well microplates, using a multichannel micropipette. The absorbance decrease was measured at $734 \mathrm{~nm}$, every $30 \mathrm{~min}$ for $8 \mathrm{~h}$, using an UV-Vis spectrophotometer (Synergy HT, Bio-Tek Instruments). The total antioxidant capacity was expressed as Trolox equivalent antioxidant capacity (in $\mu \mathrm{mol}$ of Trolox $/ \mathrm{kg}$ of coffee sample dwb). A typical calibration curve obtained under the experimental conditions used could be described by the equation: $\Delta \mathrm{Abs}_{734 \mathrm{~nm}}=-0.015+0.960 \mathrm{C}_{\text {Trolox }}\left(R^{2}=0.998\right)$, obtained with Trolox in the concentration range from 5 to $50 \mu \mathrm{mol} \mathrm{L}^{-1}$. The intrinsic absorption of the coffee extracts was measured by adding extraction solution instead of ABTS. ${ }^{+}$solution and was considered not significant because it was equivalent to less than 0.005 absorbance units. The measurement of antioxidant activity was performed in quadruplicate for each extract.

\section{Total Phenolic Content of Roasted Coffee}

The procedure used for the quantification of total phenolic compounds in the roasted coffee samples was performed as follows: $150 \mu \mathrm{L}$ aliquots of previously 20 -fold diluted coffee extract were treated with $50 \mu \mathrm{L}$ of Folin-Ciocalteu solution (3:10, $v / v)$, followed by addition of $100 \mu \mathrm{L}$ of sodium carbonate solution $(9 \%, w / v)$ in a 96 -well microplate. The absorbance during the reaction was monitored at $760 \mathrm{~nm}$ at intervals of $10 \mathrm{~min}$ for $2 \mathrm{~h}$ (Páscoa et al. 2013). The intrinsic absorbance of the sample extracts was determined by mixing the extracts with $50 \mu \mathrm{L}$ of $\mathrm{HCl}(0.6 \mathrm{M})$ and was found to be not significant. Similarly, the intrinsic absorbance of the reagent was measured by using the same volume of water, instead of the sample extract. The values obtained were considered in the subsequent calculations. The total phenolic content was expressed as gallic acid equivalent capacity (in $\mathrm{g}$ of gallic acid $/ \mathrm{kg}$ of coffee sample $\mathrm{dwb}$ ), according to the calibration curve described by the equation: $A_{760 \mathrm{~nm}}=0.072+0.065 C_{\text {gallic acid }}\left(R^{2}=0.999\right)$, obtained with gallic acid at concentrations in the range $1-20 \mathrm{mg} \mathrm{L}^{-1}$. The measurement of the total phenolic content was performed in quadruplicate for each extract.

\section{In-line NIR Spectra Acquisition}

Near-infrared spectra were acquired in diffuse reflectance mode, using a Fourier transform NIR spectrometer (FTLA 2000, ABB, Quebec, Canada) controlled with BomenGrams software (version 7, ABB, QC, Canada). The spectrometer was connected to a diffuse reflectance probe and an indium-gallium-arsenide (InGaAs) detector. The probe (SabIR, ThermoNicolet, Madison, WI), featuring a sapphire window with a $0.20-\mathrm{cm}^{2}$ illumination area, was placed outside the coffee roaster, facing the glass observation window at an approximate distance of $2 \mathrm{~mm}$. In this way, the probe is as close as possible from the coffee sample under roasting without being significantly heated (Fig. 1).

Each NIR spectrum was acquired using an average of 64 scans, resolution of $8 \mathrm{~cm}^{-1}$, and wavenumber interval from 10.000 to $4.000 \mathrm{~cm}^{-1}$. Under these conditions, approximately $30 \mathrm{~s}$ was required to record each spectrum. For each saved spectrum, a coffee sample was immediately collected to match that particular spectral analysis. A total of 56 spectra were used to develop chemometric models for antioxidants estimation. Before each roasting process, a background spectrum was obtained using a disk of PTFE (Teflon) reference material.

\section{Multivariate Data Analysis}

\section{Exploratory Data Analysis}

All chemometric calculations were performed using Matlab version 8.3 (MathWorks, Natick, MA, USA) and partial least 
Fig. 1 Laboratory experimental setup for the real-time coffee roasting monitoring with near infrared spectroscopy: 1 , diffuse reflectance probe; 2 , frontal roaster glass window; 3 , roaster's drum; 4 , sampling door; 5 , samples/ coffee beans; 6 , roaster's display; 7 , sampling door knob

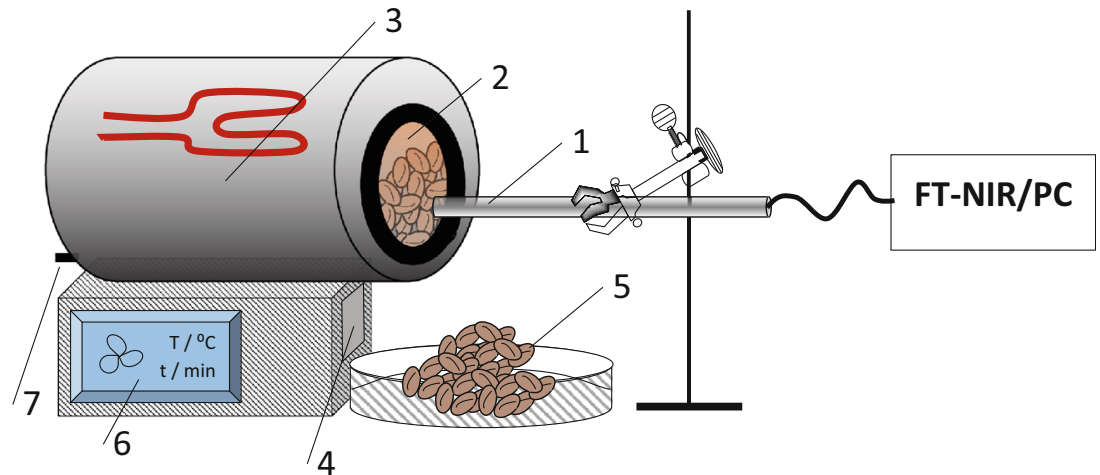

squares (PLS) Toolbox version 7.5 (Eigenvector Research Inc., Wenatchee, WA, USA).

Before applying any chemometric model, all data were subjected to mean centering.

After collection all 56 NIR spectra, a principal component analysis (PCA) (Naes et al. 2004) model was constructed to identify patterns over the roasting process and/or any outliers. The squared prediction error and Hotelling's $T^{2}$ statistics were evaluated to discard the presence of outliers. The criterion for detecting outliers was based on the comparison of vector lengths obtained for the normalized statistics divided by the 95\% confidence limits (Naes et al. 2004).

\section{PLS Modeling}

For the PLS model optimization, the spectra were preprocessed exploiting Savitzky-Golay (SG) method (different parameters including first and second derivative were evaluated), standard normal variate (SNV) transformation, and multiplicative scatter correction, individually and combined. These pre-processing methods have been extensively used in the past for similar samples measured with NIR and modeling purposes (Santos et al. 2016b; Páscoa et al. 2013; Pizarro et al. 2004). Essentially, the selected methods remove baseline variations and correct for scale differences.

The PLS method with the PLS1 algorithm (Geladi and Kowalski 1986) was used to generate the predictive models for the parameters TAC and TPC, and each parameter was modeled individually. A set of 56 spectra was used to develop the PLS models for TPC and TAC determination. This set was divided randomly into two groups (ensuring an even distribution in terms of the values obtained by the reference procedures): one group (39 samples) was used for calibration and the other group, with the remaining 17 samples, was used for independent testing. The number of latent variables (LV) was defined with the calibration set, using the leave-one-out crossvalidation method (Sarraguça and Lopes 2009). The optimization of the PLS models involved the combination of different spectral regions of the acquired NIR spectra (details in the "NIR spectral analysis" section). The accuracy of the models was evaluated in terms of the root mean square errors for calibration (RMSEC), cross-validation (RMSECV), and prediction (RMSEP) (Eq. 1).

$\operatorname{RMSE}(\mathrm{C}, \mathrm{V}, \mathrm{P})=\sqrt{\frac{\sum_{i=1}^{N}\left(y_{i}-y_{i}\right)^{2}}{N}}$

In Eq. $1, N$ is the number of samples, and $y i$ and $y_{i}$ are the experimental and NIR prediction values for sample $i$, obtained for the calibration (RMSEC), cross-validation (RMSECV), and prediction sets (RMSEP). Additional parameters used to evaluate the accuracy of the PLS models were the coefficient of determination $\left(R_{\mathrm{P}}^{2}\right)$ and the range error ratio (RER), calculated according to Eq. 2, both relative to the prediction set.

$\operatorname{RER}=(\Delta \mathrm{y}) / \mathrm{RMSEP}$

In Eq. 2, $\Delta y$ is the difference between the maximum and minimum experimental values of TAC or TFC in the independent test set. RER values above 10 indicate good predictive models (De Girolamo et al. 2009). Using the concept of net analyte signal (NAS), the figures of merit for the multivariate PLS models (limit of detection (LOD), sensitivity (SEN), and selectivity (SEL)) were determined for each modeled parameter, employing a strategy similar to the univariate approach (Sarraguça and Lopes 2009). SEN and SEL were determined using the following equations:

$\mathrm{SEL}=\left\|r^{*}\right\| /\|r\|$

$\mathrm{SEN}=\left\|s^{*}\right\|$

In the previous equations, $r$ is a sample spectra, $r^{*}$ is the NAS vector, and $s^{*}$ is the ratio between the NAS of any sample and the corresponding PLS prediction. Selectivity values were calculated by taking the average over all samples (SEL is multiplied by 100 and displayed as \%). 


\section{Results and Discussion}

\section{Antioxidant Profile during Coffee Roasting}

The ABTS colorimetric assay was employed as this methodology has been shown to be selective towards the main coffee compounds tentatively related to antioxidant activity, namely melanoidins (Delgado-Andrade et al. 2005; Pérez-Martínez et al. 2010), phenolic compounds (Andrade et al. 2012) and, particularly, within this last group, chlorogenic acids (Charurin et al. 2002; Pérez-Martínez et al. 2010). The results obtained are presented in Fig. 2.

The higher initial TAC values for the Robusta cultivar, compared to the Arabica species, may be due to the higher intrinsic content of chlorogenic acids in Robusta (ca. 11\%,w/ w) than in Arabica $(8 \%, w / w)$ (Farah 2012). An additional justification is suggested by Vignoli et al. (2011) based on the higher content of polyphenols and caffeine in Robusta species. However, to the best of our knowledge, no previous modeling or experimental studies have investigated the reaction between ABTS and caffeine.


Fig. 2 TAC profiles for different coffee samples (-Robusta India; Robusta Uganda; - Arabica Brazil; - Arabica Timor) and roasting modes (a slow roast; $\mathbf{b}$ fast roast)
With respect to the changes in antioxidant levels during roasting, in both SR and FR assays, the Robusta samples showed constant and higher TAC values, compared to the increasing profile observed for the Arabica samples. Similar studies of antioxidant activity profiles based on ABTS assays have been performed, generally based on three measurements in order to consider light, medium, and dark roast samples. Vignoli et al. (2011) found that the antioxidant activity was not affected during roasting of coffee beans for 7-10 $\mathrm{min}$ at 214-225 ${ }^{\circ} \mathrm{C}$. Delgado-Andrade et al. (2005) reported an increase in TAC from light roast to medium roast and leveling off from medium roast to dark roast. Sacchetti et al. (2009) roasted coffee samples for $10 \mathrm{~min}$ at $180{ }^{\circ} \mathrm{C}$ and found an increase up to medium roast, followed by a subsequent decrease. Nicoli et al. (1997) used 2,2'-azobis (2amidinopropane) dihydrochloride as the peroxyl radical generator, instead of ABTS, in a roasting experiment with four samples obtained between 8 and 20 min (using a roasting temperature of ca. $200{ }^{\circ} \mathrm{C}$ ), and found higher antioxidant concentrations in medium and dark roast Arabica samples.

There are several factors that make it difficult to directly compare the TAC profile results described in the literature. These include the intrinsic heterogeneity of coffee samples, the roasting procedure (which may be performed within 3 min (Del Castillo et al. 2002) or for up to ca. $20 \mathrm{~min}$ (Nicoli et al. 1997), at fixed or increasing temperature), and the analytical procedure employed for antioxidant activity measurement. In the last case, differences in extraction conditions, such as the medium used, temperature, and time, can lead to different extraction yields.

Possible explanations for the present findings include the existence of antioxidant compound precursors in Arabica beans and/or the different antioxidant activities of different compounds. The antioxidant activity profile during the roasting process reflects the sum of the contributions of all the antioxidant compounds present at a given moment during the roasting process. It is widely accepted that the concentration of chlorogenic acids decreases during the roasting process, while the concentration of melanoidins increases. The changes in the concentrations of these compounds during roasting could be largely responsible for the antioxidant profile.

The overall effect of using different roasting experimental conditions did not appear to be significant, since similar profiles were obtained for the Robusta and Arabica species in the FR and SR assays. In similar roasting assays used for in-line monitoring of sucrose (Santos et al. 2016b) and titratable acidity (Santos et al. 2016a), the influence of different roasting conditions was much more noticeable. In the present case, the evidence suggested that there were similar changes in the kinetics of the antioxidant compound degradation and formation reactions under the different roasting conditions used.

For both FR and SR procedures, the final TAC values for the Robusta and Arabica coffees were mostly similar (with the 
exception of the Timor Arabica sample in the SR assay), which could have been due to the typically similar melanoiidin concentrations in both species after roasting (Farah 2012). The TAC values obtained for the roasted coffees were within the range reported previously (Delgado-Andrade et al. 2005; Vignoli et al. 2011).

\section{Folin-Ciocalteu Profile during Coffee Roasting}

The widely used Folin-Ciocalteu assay for the determination of phenolic compounds was adopted. The profiles obtained in this procedure were similar to those previously observed in the ABTS radical scavenging assays (Fig. 3). This was expected, because the Folin-Ciocalteu reagent is selective to both chlorogenic acids and melanoidins (Pérez-Martínez et al. 2010), and the reaction involves similar chemical processes (based on electron transfer) as the ABTS radical scavenging technique (Huang et al. 2005).

For the Robusta samples, the TPC values remained approximately constant and were within the same range for the SR and FR roasting processes. For the Arabica samples, similar
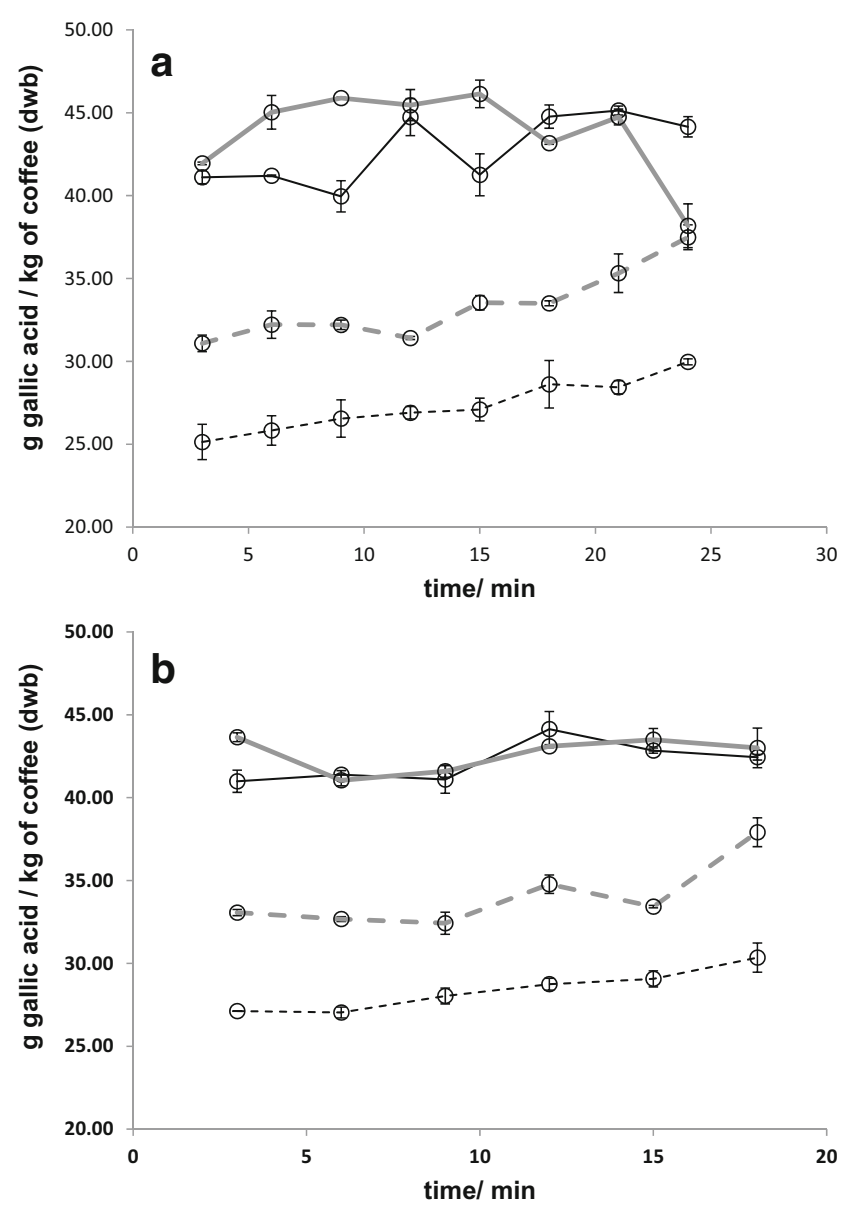

Fig. 3 TPC profiles for different coffee samples (-Robusta India; - Robusta Uganda; - - Arabica Brazil; — - Arabica Timor) and roasting modes (a slow roast; $\mathbf{b}$ fast roast) increases in TPC values were observed during the two roasting processes. However, these increases were lower than those observed in the TAC assays; this was the most notable difference between the ABTS and Folin-Ciocalteu results. The associations previously identified, concerning the initial and final TAC values and the typical intrinsic composition of green and roasted coffee, could also be applied to the results obtained using the Folin-Ciocalteu technique. The profiles observed for the Arabica samples showed that although the phenolic fraction was consumed during the roasting procedure, there was a smaller increase of the TPC values, compared to TAC, which could be explained by the lower selectivity of the Folin-Ciocalteu technique towards melanoidins (Pérez-Martínez et al. 2010). In addition, the profile differences could be related to the reaction yields for phenolics and melanoidins achieved with the Folin-Ciocalteu and ABTS methods (no specific data are available to support this possibility). Pérez-Martínez et al. (2010) reported that the Folin-Ciocalteu procedure may not be the most appropriate methodology for the measurement of phenolics in coffee samples and instead suggested the use of electron spin resonance (ESR) spectroscopy. The values obtained here for TPC in the roasted coffee were of the same magnitude as those found in other studies (Morais et al. 2009; Stelmach et al. 2015; PérezMartínez et al. 2010). However, different profiles have been described in the literature. Morais et al. (2009) roasted Robusta samples at $190 \pm 10{ }^{\circ} \mathrm{C}$ for three different periods $(6,8$, and $10 \mathrm{~min})$ and observed decreases during the roasting processes. Vignoli et al. (2011), using both Arabica and Robusta samples, concluded that no clear trends could be found for TPC values during the roasting process.

As observed in the ABTS assays, there were no significant differences between FR and SR in terms of the phenolics profile. The same conclusions reached for the ABTS results could also be applied to the Folin-Ciocalteu assays.

\section{NIR Spectral Analysis}

The NIR spectra acquired during the roasting assays were similar to the spectra obtained in previous studies (Santos et al. 2016a; Santos et al. 2016b). The main changes in the spectra of the roasting process were an absorbance decrease in the water band region (5200-5000 $\mathrm{cm}^{-1}$ ) due to moisture loss and an absorbance increase in the combination band region (5000-4000 $\mathrm{cm}^{-1}$ ) (Fig. 4). A more in-depth discussion concerning the main wavelength intervals and their correlations with chemical and physical changes in the coffee during the roasting process has been provided elsewhere (Santos et al. 2016a).

The NIR spectral range was divided into five regions in order to identify those that showed the greatest association with the antioxidant properties. Region $1(10,000$ $7300 \mathrm{~cm}^{-1}$ ) is mainly related to physical properties (bean size, 


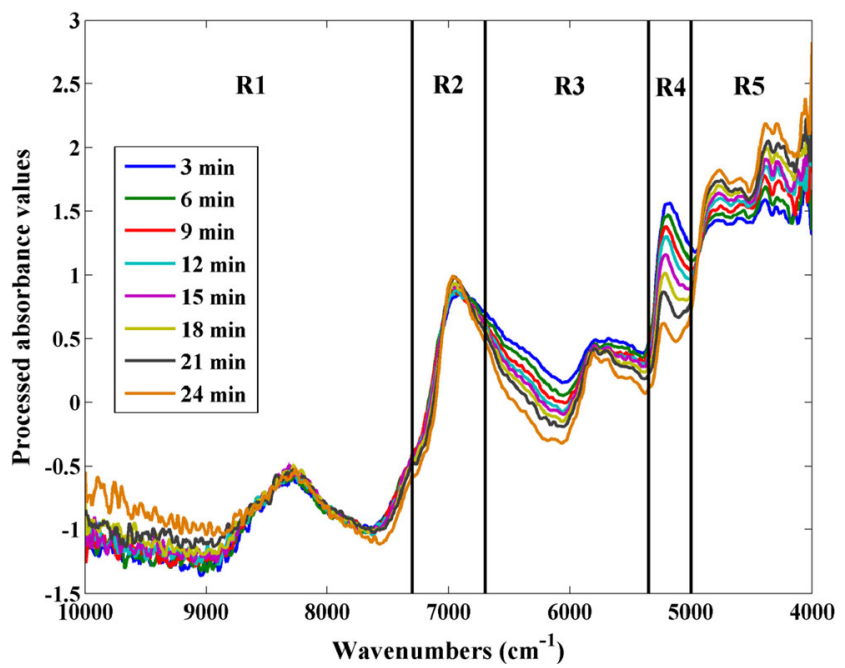

Fig. 4 Near-infrared spectra collected during a slow roast procedure of Robusta India coffee beans, indicating the selected five spectral regions: $R 1,10,000-7300 \mathrm{~cm}^{-1} ; R 2,7299-6700 \mathrm{~cm}^{-1} ; R 3,6699-5350 \mathrm{~cm}^{-1} ; R 4$, $5349-5000 \mathrm{~cm}^{-1}$; and $R 5,4999-4000 \mathrm{~cm}^{-1}$

shape and density); region $2\left(7299-6700 \mathrm{~cm}^{-1}\right)$ is mostly affected by the water content; region $3\left(6699-5400 \mathrm{~cm}^{-1}\right)$ essentially captures chemical information concerning $\mathrm{C}-\mathrm{H}$ and S-H bonds; region $4\left(5399-5000 \mathrm{~cm}^{-1}\right)$ includes the first overtone and combination band regions and is most affected by $\mathrm{RCO}_{2} \mathrm{~N}, \mathrm{CONH}_{2}$, and $\mathrm{H}_{2} \mathrm{O}$ vibrations; and region 5 (4999$4000 \mathrm{~cm}^{-1}$ ) essentially contains chemical information related to $\mathrm{C}-\mathrm{H}, \mathrm{N}-\mathrm{H}$, and $\mathrm{C}-\mathrm{O}$ vibrations.

The PCA developed model revealed that no outliers were present in the set of 56 spectra. Additionally, the model scores suggest the existence of four clusters each including a single coffee variety and origin, irrespective of the degree of roast (Supplementary Data, Fig. SD1). These results were similar to previous findings (Santos et al. 2016a).

\section{PLS Model Testing}

In order to optimize the PLS models, in terms of spectral regions, different combinations of the identified wavelength intervals were tested, and to each one, the referred preprocessing strategies were applied. Models for TAC and TPC were optimized based on a compromise between the numbers of LVs and RMSECV (Table 1). The best spectral window for both models was obtained using a combination of the R3 (6699-5400 $\left.\mathrm{cm}^{-1}\right)$ and R5 $\left(4999-4000 \mathrm{~cm}^{-1}\right)$ regions, pre-processed with SG using a 15-point filter width, a secondorder polynomial, first derivative, and SNV transformation. During the roasting process, there was a general absorbance decrease in region R3 (especially within the interval 6300 $6000 \mathrm{~cm}^{-1}$ ). In region $\mathrm{R} 5$, the absorbance was roughly constant within the interval $5000-4500 \mathrm{~cm}^{-1}$, while a significant absorbance increase occurred at $4300-4100 \mathrm{~cm}^{-1}$.

Further evaluation of the PLS models was performed using the independent set. The parameters RMSEP, RER, and $R_{\mathrm{P}}{ }^{2}$ were determined for the two PLS models (Table 1 and Fig. 5).

Selectivity values around $12-20 \%$, or the spectra fraction captured by the NAS vector unique to the analyte of interest, showed that a significant part of the signal was employed in model construction. The sensitivity can be understood as the slope of the calibration curve (Páscoa et al. 2013). The sensitivity values can be compared when the same analyte is being determined by different models. In this work, two different properties are being estimated and, therefore, the comparison between sensitivities does not provide a useful interpretation. The LOD values demonstrated that the developed technique was suitable for the real-time control of antioxidant properties.

The analysis to the regression coefficient vectors obtained for both PLS methods showed relevance of the identified regions but did not identify specific wave numbers (or more restricted wave number intervals) (Supplementary Data, Fig. SD2). In light of the present data and findings reported in the literature, an attempt was made to correlate the spectral changes with potential antioxidant compounds. The absorbance decrease in the $\mathrm{R} 3$ region (assigned to $\mathrm{O}-\mathrm{H}$ and $\mathrm{N}-\mathrm{H}$ (first overtone), $\mathrm{C}=\mathrm{O}$ (second overtone), $\mathrm{C}-\mathrm{H}, \mathrm{Ar}-\mathrm{CH}$, and $\mathrm{N}$ $\mathrm{H}$ bonds) could be attributed to a decrease in the concentration of aryl substituent compounds, notably phenolic compounds and chlorogenic acids (Ribeiro et al. 2011). The absorbance

Table 1 Figures of merit for the PLS calibration models for the different chemical methods using the optimized spectral windows (6699-5400 and $4999-4000 \mathrm{~cm}^{-1}$ )

\begin{tabular}{|c|c|c|c|c|c|c|c|c|c|}
\hline \multirow[t]{2}{*}{ Antioxidant assay } & \multicolumn{3}{|c|}{ Calibration set } & \multicolumn{3}{|c|}{ Independent set } & \multirow[t]{2}{*}{ Sensitivity $^{\mathrm{b}}$} & \multirow[t]{2}{*}{ Selectivity (\%) } & \multirow[t]{2}{*}{$\mathrm{LOD}^{\mathrm{c}}$} \\
\hline & LV & RMSEC $^{\mathrm{a}}$ & RMSECV $^{a}$ & RMSEP $^{\text {a }}$ & $R_{\mathrm{P}}^{2}$ & RER & & & \\
\hline ABTS & 3 & 75.7 & 119 & 94.8 & 0.909 & 10.4 & 0.01 & 0.20 & 284 \\
\hline Folin-Ciocalteu & 4 & 0.394 & 2.28 & 1.97 & 0.927 & 10.7 & 0.14 & 0.12 & 5.91 \\
\hline
\end{tabular}

${ }^{a}$ Root mean square error expressed in mmol Trolox/kg of coffee (dwb) and g of gallic acid/kg of coffee (dwb) for ABTS and Folin-Ciocalteu assays, respectively

${ }^{\mathrm{b}}$ Sensitivity values expressed as spectral units/concentration units

${ }^{\mathrm{c}}$ Limit of detection units expressed in mmol Trolox/kg of coffee (dwb) and g of gallic acid/kg of coffee (dwb) for ABTS and Folin-Ciocalteu assays, respectively 

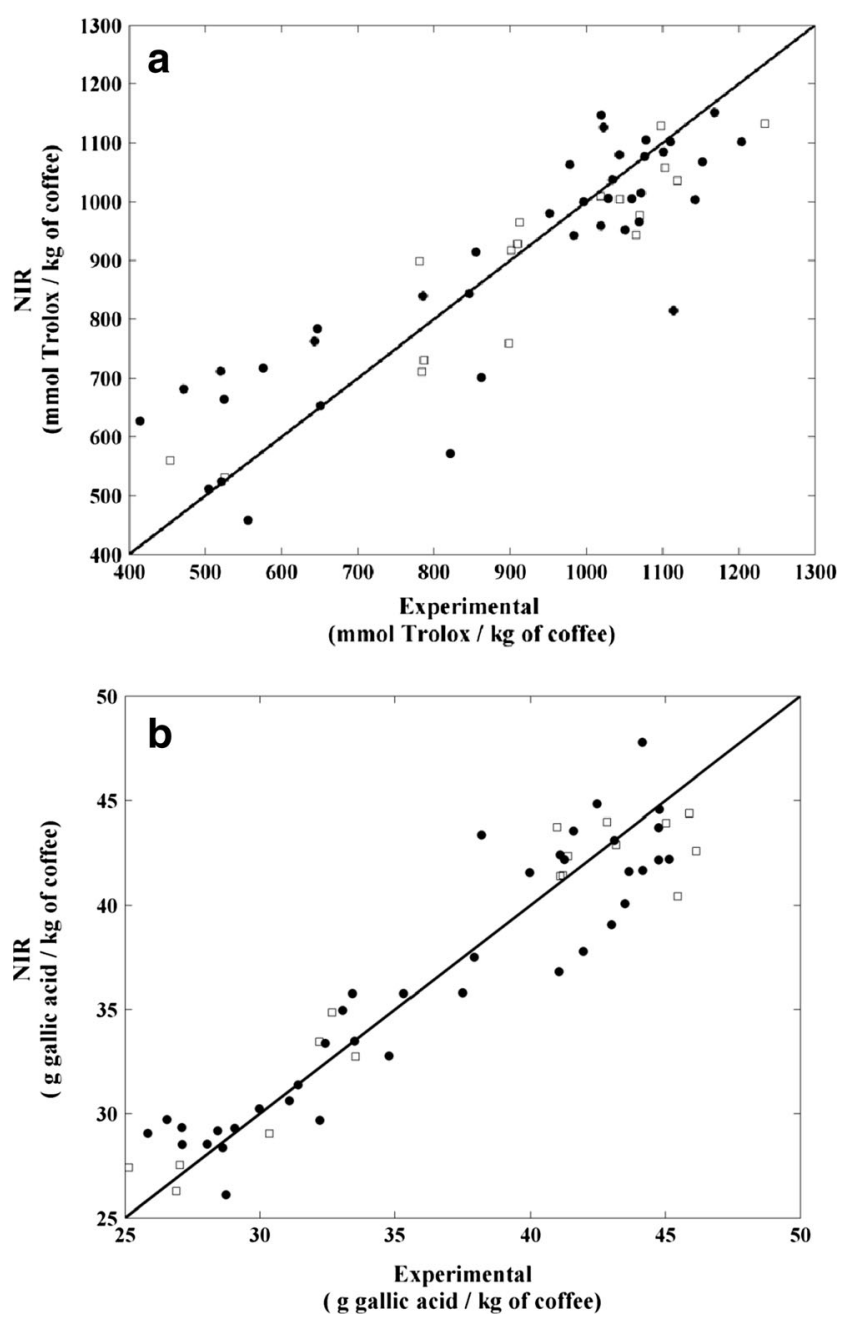

Fig. 5 Comparison between wet antioxidant assay results and PLS crossvalidation predictions for a TAC and b TPC: calibration set (filled circles); prediction set (open square)

increase in the $\mathrm{R} 5$ region at $4300-4100 \mathrm{~cm}^{-1}$ (assigned to $\mathrm{O}-\mathrm{H}$ and $\mathrm{N}-\mathrm{H}$ combination bands, $\mathrm{C}-\mathrm{H}$ and $\mathrm{C}-\mathrm{H}$ combination bands, and $\mathrm{C}-\mathrm{H}$ and $\mathrm{C}-\mathrm{C}$ combination bands) could be attributed to the formation of melanoidins (a complex mixture consisting mainly of carbohydrates and amino acids formed during the roasting procedure, together with low levels of phenolic compounds and chlorogenic acids) (Preedy 2015). These correlations are in accordance with the main chemical drivers previously associated with the antioxidant profile.

\section{Conclusions}

The present work demonstrates the capacity of NIR spectroscopy for real-time evaluation of the antioxidant potential of coffee during the coffee bean roasting process. Different coffee species and origins were tested, considering the influence of the roasting process on the total phenolic content and the total antioxidant capacity.
PLS regression models for TAC and TPC yielded RER values above 10 , indicative of acceptable predictive ability in practical applications. Prediction errors were of the same order of magnitude as for wet chemistry reference methodologies used to determine TAC and TPC. A significant part of the spectrum was employed for construction of the models (6699-5400 and 4999-4000 $\mathrm{cm}^{-1}$ ), resulting in selectivity values between 12 and $20 \%$.

The values found for TAC and TPC in green coffee beans were in good agreement with previous reports. The profiles obtained here contribute to the current discussion, where it has been difficult to reach a consensus. The methodology developed is much faster and simpler to perform, compared to the usual batch methodologies, and offers a valuable analytical tool for the prediction of antioxidant activity in coffee samples or during the roasting procedure. It also assists in understanding the main chemical processes that govern the antioxidant activity. Additionally, there were no obvious effects of roasting speed on the antioxidant profiles of the coffees in the assays performed here.

The presented strategy is especially attractive for use in the coffee industry due to the easy setup (the roasting process can be monitored through the glass window of a roaster) and the intrinsic analytical advantages of NIRS, which is a nondestructive and non-invasive technique that is simple to operate.

Acknowledgements T.A. Catelani is grateful to CAPES for a scholarship (PDSE no. 99999.000655/2015-05) and to CNPq. R.N.J. Páscoa acknowledges financial support from SFRH/BPD/81384/2011. This work received financial support from FCT/MEC through national funds and was co-financed by FEDER under partnership agreement PT2020 UID/QUI/50006/2013 - POCI/01/0145/FEDER/007265. The authors also acknowledge funding from NORTE-01-0145-FEDER-000011 and iMed.ULisboa (grant UID/DTP/04138/2013).

\section{References}

Alves, R. C., Almeida, I. M. C., Casal, S., \& Oliveira, M. B. P. P. (2010). Isoflavones in coffee: influence of species, roast degree, and brewing method. Journal of Agricultural and Food Chemistry, 58(5), 3002-3007. doi:10.1021/j9039205.

Andrade, K. S., Gonçalvez, R. T., Maraschin, M., Ribeiro-Do-Valle, R. M., Martínez, J., \& Ferreira, S. R. S. (2012). Supercritical fluid extraction from spent coffee grounds and coffee husks: antioxidant activity and effect of operational variables on extract composition. Talanta, 88, 544-552. doi:10.1016/j.talanta.2011.11.031.

Borrelli, R. C., Visconti, A., Mennella, C., Anese, M., \& Fogliano, V. (2002). Chemical characterization and antioxidant properties of coffee melanoidins. Journal of Agricultural and Food Chemistry, 50(22), 6527-6533. doi:10.1021/jf025686o.

Budryn, G., Pałecz, B., Rachwał-Rosiak, D., Oracz, J., Zaczyńska, D., Belica, S., et al. (2015). Effect of inclusion of hydroxycinnamic and chlorogenic acids from green coffee bean in $\beta$-cyclodextrin on their interactions with whey, egg white and soy protein isolates. Food Chemistry, 168, 276-287. doi:10.1016/j.foodchem.2014.07.056. 
Carlsen, M. H., Halvorsen, B. L., Holte, K., Bøhn, S. K., Dragland, S., Sampson, L., et al. (2010). The total antioxidant content of more than 3100 foods, beverages, spices, herbs and supplements used worldwide. Nutrition Journal, 9, 3. doi:10.1186/1475-2891-9-3.

Charurin, P., Ames, J. M., \& Del Castillo, M. D. (2002). Antioxidant activity of coffee model systems. Journal of Agricultural and Food Chemistry, 50(13), 3751-3756. doi:10.1021/jf011703i.

De Girolamo, A., Lippolis, V., Nordkvist, E., \& Visconti, A. (2009). Rapid and non-invasive analysis of deoxynivalenol in durum and common wheat by Fourier-transform near infrared (FT-NIR) spectroscopy. Food Additives \& Contaminants: Part A, 26(6), 907-917. doi:10.1080/02652030902788946.

Del Castillo, M. D., Ames, J. M., \& Gordon, M. H. (2002). Effect of roasting on the antioxidant activity of coffee brews. Journal of Agricultural and Food Chemistry, 50(13), 3698-3703. doi:10.1021/jf011702q.

Delgado-Andrade, C., Rufián-Henares, J. A., \& Morales, F. J. (2005). Assessing the antioxidant activity of melanoidins from coffee brews by different antioxidant methods. Journal of Agricultural and Food Chemistry, 53(20), 7832-7836. doi:10.1021/jf0512353.

Devasagayam, T. P. A., Kamat, J. P., Mohan, H., \& Kesavan, P. C. (1996). Caffeine as an antioxidant: inhibition of lipid peroxidation induced by reactive oxygen species. Biochimica et Biophysica Acta, 1282, 63-70.

Esteban-Díez, I., González-Sáiz, J. M., \& Pizarro, C. (2004). Prediction of sensory properties of espresso from roasted coffee samples by near-infrared spectroscopy. Analytica Chimica Acta, 525(2), 171182. doi:10.1016/j.aca.2004.08.057.

Esteban-Díez, I., González-Sáiz, J. M., Sáenz-González, C., \& Pizarro, C. (2007). Coffee varietal differentiation based on near infrared spectroscopy. Talanta, 71(1), 221-229. doi:10.1016/j. talanta.2006.03.052.

Farah, A. (2012). Coffee constituents. In Y.-F. Chu (Ed.), Coffee: emerging health effects and disease prevention (pp. 21-58). Blackwell Publishing Ltd. doi:10.1002/9781119949893.ch2.

Gallardo, C., Jiménez, L., \& García-Conesa, M.-T. (2006). Hydroxycinnamic acid composition and in vitro antioxidant activity of selected grain fractions. Food Chemistry, 99(3), 455-463. doi:10.1016/j.foodchem.2005.07.053.

Geladi, P., \& Kowalski, B. R. (1986). Partial least-squares regression-a tutorial. Analytica Chimica Acta, 185, 1-17.

Gökmen, V., Serpen, A., \& Fogliano, V. (2009). Direct measurement of the total antioxidant capacity of foods: the "QUENCHER" approach. Trends in Food Science \& Technology, 20, 278-288. doi:10.1016/j.jcs.2008.06.002.

Huang, D., Ou, B., \& Prior, R. L. (2005). The chemistry behind antioxidant capacity assays. Journal of Agricultural and Food Chemistry, 53(6), 1841-1856.

ISO (11294:1994) Roasted ground coffee - Determination of moisture content - Method by determination of loss in mass at 103 degrees $\mathrm{C}$ (Routine method).

Lee, J., Koo, N., \& Min, D. B. (2004). Reactive oxygen species, aging, and antioxidative nutraceuticals. Comprehensive Reviews in Food Science and Food Safety, 3(1), 21-33. doi:10.1111/j.15414337.2004.tb00058.x.

Morais, S. A. L., Aquino, F. J. T., Nascimento, P. M., Nascimento, E. A., \& Chang, R. (2009). Compostos bioativos e atividade antioxidante do café conilon submetido a diferentes graus de torra. Química Nova, 32(2), 327-331.

Moreira, D. P., Monteiro, M. C., Ribeiro-Alves, M., Donangelo, C. M., \& Trugo, L. C. (2005). Contribution of chlorogenic acids to the ironreducing activity of coffee beverages. Journal of Agricultural and Food Chemistry, 53(5), 1399-1402. doi:10.1021/jf0485436.

Mussatto, S. I., Ballesteros, L. F., Martins, S., \& Teixeira, J. A. (2011). Extraction of antioxidant phenolic compounds from spent coffee grounds. Separation and Purification Technology, 83(1), 173-179. doi:10.1016/j.seppur.2011.09.036.
Naes, T., Isakson, T., Fearn, T., \& Davies, T. (2004). Interpreting PCR and PLS solutions. In a user-friendly guide to multivariate calibration and classification. Chichester: NIR publications.

Nicoli, M. C., Anese, M., Manzocco, L., \& Lerici, C. R. (1997). Antioxidant properties of coffee brews in relation to the roasting degree. Food Science and Technology-Lebensmittel-Wissenschaft \& Technologie, 30, 292-297. doi:10.1006/fstl.1996.0181.

Páscoa, R. N. M. J., Magalhães, L. M., \& Lopes, J. A. (2013). FT-NIR spectroscopy as a tool for valorization of spent coffee grounds: application to assessment of antioxidant properties. Food Research International, 51(2), 579-586. doi:10.1016/j.foodres.2013.01.035.

Pérez-Jiménez, J., \& Torres, J. L. (2011). Analysis of nonextractable phenolic compounds in foods: the current state of the art. Journal of Agricultural and Food Chemistry, 59(24), 12713-12724. doi:10.1021/jf203372w.

Pérez-Martínez, M., Caemmerer, B., De Peña, M. P., Concepción, C., \& Kroh, L. W. (2010). Influence of brewing method and acidity regulators on the antioxidant capacity of coffee brews. Journal of Agricultural and Food Chemistry, 58(5), 2958-2965. doi:10.1021 /j90037375.

Pizarro, C., Esteban-Díez, I., González-Sáiz, J. M., \& Forina, M. (2007). Use of near-infrared spectroscopy and feature selection techniques for predicting the caffeine content and roasting color in roasted coffees. Journal of Agricultural and Food Chemistry, 55(18), 74777488. doi:10.1021/jf071139x.

Pizarro, C., Esteban-Díez, I., Nistal, A. J., \& González-Sáiz, J. M. (2004). Influence of data pre-processing on the quantitative determination of the ash content and lipids in roasted coffee by near infrared spectroscopy. Analytica Chimica Acta, 509(2), 217-227. doi:10.1016/j. aca.2003.11.008.

Preedy, V. R. (Ed.) (2015). Coffee in health and disease. Amsterdam: Academic.

Ribeiro, J. S., Ferreira, M. M. C., \& Salva, T. J. G. (2011). Chemometric models for the quantitative descriptive sensory analysis of Arabica coffee beverages using near infrared spectroscopy. Talanta, 83(5), 1352-1358. doi:10.1016/j.talanta.2010.11.001.

Sacchetti, G., Di Mattia, C., Pittia, P., \& Mastrocola, D. (2009). Effect of roasting degree, equivalent thermal effect and coffee type on the radical scavenging activity of coffee brews and their phenolic fraction. Journal of Food Engineering, 90(1), 74-80. doi:10.1016/j. jfoodeng.2008.06.005.

Sánchez-González, I., Jiménez-Escrig, A., \& Saura-Calixto, F. (2005). In vitro antioxidant activity of coffees brewed using different procedures (Italian, espresso and filter). Food Chemistry, 90(1-2), 133139. doi:10.1016/j.foodchem.2004.03.037.

Santos, J. R., Lopo, M., Rangel, A. O. S. S., \& Lopes, J. A. (2016a). Exploiting near infrared spectroscopy as an analytical tool for online monitoring of acidity during coffee roasting. Food Control, 60, 408-415. doi:10.1016/j.foodcont.2015.08.007.

Santos, J. R., Viegas, O., Páscoa, R. N. M. J., Ferreira, I. M. P. L. V. O., Rangel, A. O. S. S., \& Lopes, J. A. (2016b). In-line monitoring of the coffee roasting process with near infrared spectroscopy: measurement of sucrose and colour. Food Chemistry, 208, 103-110. doi:10.1016/j.foodchem.2016.03.114.

Sarraguça, M. C., \& Lopes, J. A. (2009). The use of net analyte signal (NAS) in near infrared spectroscopy pharmaceutical applications: interpretability and figures of merit. Analytica Chimica Acta, 642(1-2), 179-185. doi:10.1016/j.aca.2008.10.006.

Stelmach, E., Pohl, P., \& Szymczycha-Madeja, A. (2015). The content of Ca, $\mathrm{Cu}, \mathrm{Fe}, \mathrm{Mg}$ and $\mathrm{Mn}$ and antioxidant activity of green coffee brews. Food Chemistry, 182, 302-308. doi:10.1016/j.foodchem.2015.02.105.

Vignoli, J. A., Bassoli, D. G., \& Benassi, M. T. (2011). Antioxidant activity, polyphenols, caffeine and melanoidins in soluble coffee: the influence of processing conditions and raw material. Food Chemistry, 124(3), 863-868. doi:10.1016/j.foodchem.2010.07.008. 